Age ranged from 18 to 59 years old, and the prevalence was higher in women aged 25 to 39 (14/3.9\% CI 56.99-67.01), from Teresina (23/With no partner (26/7.2\%, CI 92.2696.94), with less than eight years of education at school (19.5\% and 5.4\%, CI 73.07-81.73). IC 63.38-73.02), catholic (19/5.3\%, IC 75.97-84.23), income less than 1 minimum national wage $(14 / 3.9 \%$, CI $39.85-50,15)$ and acting in pubs (14/3.9\%, IC 45.63-55.97).

Conclusion High prevalence of syphilis among the younger sex workers, unmarried, catholic, impoverished, poorly schooled and working indoors. It is necessary to reinforce intervention measures, focusing on health education, in order to reduce the chain of transmission and safer sexual practices.

\section{LB3.253 SPATIAL DISTRIBUTION PATTERN ANALYSIS ON SYPHILIS IN CHINA}

Xiangdong Gong, Peixuan Men, Xiaoli Yue, Xiaohong Su, Juan Jiang. Institute of Dermatology, Chinese Academy of Medical Sciences and Peking Union Medical College

\subsection{6/sextrans-2017-053264.488}

Introduction In past twenty years, incidence of syphilis has been increasing and remained a public issue in China. In order to precisely control syphilis epidemic in the country, it was very important to explore and understand the spatial distribution pattern of syphilis in county level in China.

Methods We used the software of ArcGIS(version 10) to set up the national geographic information system(GIS) database of syphilis of all counties in China in 2015. The exploratory spatial data analysis(ESDA), including frequency, the global and local spatial autocorrelation methods were used to explore the spatial distribution characteristics of syphilis.

Results The median of incidence of syphilis was 26.05/ 100,000 population ( $25 \%$ percentile and $75 \%$ percentile were 15.70 and 44.77/100,000 population, respectively), minimum with 0.84 , maximum with $433.28 / 100,000$ population in all 2925 counties of Chinese mainland in 2015, and appeared significant positive skewing (skewness was 3.19). The global trend analysis indicated that the whole distribution of syphilis from northwest to southeast appeared as "U" shape and suggested that the areas of high incidence of syphilis located in northwest and southeast coastal regions in China. The global spatial autocorrelation analysis showed a positive spatial autocorrelation (Global Moran's I is 0.5962, p<0.001) and a high-high aggregation model (General G is 0.0441, p<0.001) for syphilis epidemics in county-level in China. The local spatial autocorrelation analysis was further used to output the hot-spot mapping of syphilis, including primary and secondary syphilis, latent syphilis in county-level, and 117 hot-spots counties with high incidence of primary and secondary syphilis were recognised and identified, mainly distributed in Yangtze River delta, north-west, north-eastern and south China.

Conclusion GIS and exploratory spatial data analysis, with the advantages of visualisation and accurate location, should be as an important tool to apply in syphilis surveillance and control.

\section{LB3.254 GREATER THAN TENFOLD INCREASE IN PHARYNGEAL CHLAMYDIA TRACHOMATIS AMONG GAY AND BISEXUAL MEN ATTENDING AUSTRALIAN SEXUAL HEALTH CLINICS 2010-2016}

${ }^{1}$ David J Templeton, ${ }^{2}$ Nicholas B Comninos, ${ }^{3}$ Alison Rutherford, ${ }^{1}$ Sian L Goddard, ${ }^{4}$ Rebecca Guy, ${ }^{5}$ Basil Donovan, ${ }^{6}$ Rajesh Varma, ${ }^{4}$ Denton Callander. ${ }^{1}$ RPA Sexual Health, Sydney Local Health District and Kirby Institute, Unsw Australia, Sydney, Australia; ${ }^{2}$ RPA Sexual Health, Sydney Local Health District, Sydney, Australia; ${ }^{3}$ Department of Public Health And Community Medicine, Unsw Australia, Sydney, Australia; ${ }^{4}$ Kirby Institute, Unsw Australia, Sydney, Australia; ${ }^{5}$ Sydney Sexual Health Centre And Kirby Institute, Unsw Australia, Sydney, Australia; ${ }^{6}$ Sydney Sexual Health Centre, Sydney, Australia

\subsection{6/sextrans-2017-053264.489}

Introduction Gay and bisexual men (GBM) are disproportionately affected by Chlamydia trachomatis (CT). A steady increase in CT notifications has been observed in Australia's most populous state (NSW), with an increasing proportion being pharyngeal CT (PCT) notifications. Our aim was to investigate temporal trends and associated behavioural factors of PCT compared to anogenital CT among GBM attending NSW sexual health clinics.

Methods Clinical data were extracted in early 2017 for the period 2010-2016 from 26 NSW clinics. Positive test yield ("yield") was defined as the proportion of testing occasions where CT was detected.

Results 23,423 GBM were tested on 84385 occasions during the 7 year study period to end 2016. Yield of genital testing was $8.8 \%$ (95\%CI: $8.5-9.0)$, increasing by $17 \%$ over the study period $(8.1 \%-9.5 \%, \mathrm{p}<0.001)$. Yield of anorectal testing was $7.2 \%$ (95\% CI: 7.0-7.4), increasing by $23 \%$ during the study period $(6.0 \%-7.4 \% \%, \mathrm{p}<0.001)$. Yield of pharyngeal testing was $2.2 \%(95 \% \mathrm{CI}: 2.1-2.3)$, increasing by over $1200 \%$ from $0.3 \%$ in 2010 to $3.7 \%$ in $2016(\mathrm{p}<0.001)$. Of the 8933 positive CT tests (at any anatomical site), 424 (4.8\%) occurred without concurrent anogenital CT (“isolated PCT"). The proportion of isolated PCT increased from $2.0 \%$ in 2010, to $6.4 \%$ in 2015 and $4.4 \%$ in 2016 ( $\mathrm{p}<0.001$ ). In 2016, PCT was associated with injecting drug use $(p=0.014)$ and higher numbers of sexual partners $(p<0.001)$, after adjusting for men who reported symptoms or were CT contacts. These factors were the same as those associated with anogenital CT infections.

Conclusion The likelihood of detecting CT among GBM has increased over time with by far the greatest increase in positive test yield occurring in the pharynx. The same factors were associated with CT detection at all sites. Given that most untreated PCT persists on average for 2 years, is readily transmitted to anogenital sites and that one in 20 of all CT infections would be missed, consideration should be given to routine screening of the pharynx in other countries to reduce CT transmission among GBM.

\section{LB3.255 HIV PREVALENCE IN HOMELESS PEOPLE IN A NORTHEAST CAPITAL OF BRAZIL}

Braulio Vieira de Sousa Borges; Giselle Mary Ibiapina Brito; Matheus Sousa Marques Carvalho; Hugo Leonardo Barros de Ârea Leão; Layze Braz de Oliveira; Rosilane de Lima Brito Magalhães. Universidade Federal Do Piauí, Teresina - Pl, Brazil

\subsection{6/sextrans-2017-053264.490}

Introduction The Acquired Human Immunodeficiency Syndrome is one of the main public health problems in Brazil 
and in the world. The HIV epidemic is concentrated in major urban centres, with high prevalence among the vulnerable population, such as those living on the streets. Studies show that in the last two years, the number of people living with HIV and being on antiretroviral therapy increased about one third, reaching 17.0 million people, 2 million more than the 15 million in 2015. The main goal was to estimate the prevalence of HIV in homeless people.

Methods A cross-sectional study, developed at the Specialised Reference Centre for Homeless People (POP Centre), in the city of Teresina-PI, where participants were recruited using the Snowball technique. The data were collected through a form and a fast HIV test, from August to December/2016. This study comply with the ethical requirements, and had a report of the Research Ethics Committee of the Federal University of Piauí with number $1,755,893$.

Results HIV prevalence $(\mathrm{n}=112)$ was $7.1 \%$. The age ranged from 19 to over 45 years old, and the prevalence was highest at the age of 25 to 35 years old (2.6\%). Of the total number of infected individuals, the participants were single $(5.3 \%)$, brown (4.5\%), had at least one child (2.6\%), had their first sexual intercourse between 13 and 19 years old (3.5\%), have casual partnership $(2.6 \%)$ and have no monogamous partnership (3.5\%). Regarding to the use of condom with the aforementioned partnership, it was reported as sometimes $(0.8 \%)$ and $(5.3 \%)$ reported use of illicit drugs.

Conclusion The prevalence of HIV is high in people living on the street with higher incidence in singles, with early sexual activity, not having a monogamous partnership and use of illicit drugs. It is essential to trace cases in this type of population and control the follow-up of detected cases. In addition, guidance on preventive measures.

\section{LB3.256 SURVEY OF HEPATITIS B VACCINE ISSUE IN HOMELESS PEOPLE IN THE CENTRAL AREA OF A NORTHEAST CAPITAL OF BRAZIL}

Rosilane de Lima Brito Magalhães; Vanessa Moura Carvalho de Oliveira; Rômulo Veloso Nunes; Polyanna Maria Oliveira Martins; Marcos André Siqueira de Sousa; Braulio Vieira de Sousa Borges. Universidade Federal Do Piauí, Teresina - PI, Brazil

\subsection{6/sextrans-2017-053264.491}

Introduction The prevalence of hepatitis B is higher in populations at risk, such as people living on the street. This population is more vulnerable to several diseases, such as hepatitis B, which can be prevented by adhering to the three-dose schedule of hepatitis B vaccine. The main goal was to investigate the vaccination situation against hepatitis $B$ in this kind of population.

Methods Cross-sectional study with quantitative approach. The research was developed at the Specialised Reference Centre for Homeless People (POP Centre). Participants were recruited through the Snowball Technique. The data were collected through the application of a form, from September to December 2016. This study was submitted and approved by the Research Ethics Committee of the Federal University of Piauí with the number of report $1,755,893$.

Results Of the 58 participants, the majority, 49 (84.5\%) were males. The age ranged from 21 to over 50 years old. Regarding sexual behaviour, 7 (12.1\%) participants reported feeling same-sex attraction. Only $3(5.2 \%)$ reported having a monogamous partner, and among these, 2 (3.4\%) reported not using condoms with the aforementioned partner. Only 11 (19.0\%) participants had a vaccine card. Of the total, $35(60.3 \%)$ did not know how to report their vaccination status. Of the doses of hepatitis B vaccine given previously, 3 (5.2\%) reported having taken two doses of vaccine. The remainder, 15 (35.9\%), had not taken any dose of the vaccine. Only 3 (5.2\%) reported having completed the vaccination schedule.

Conclusion Considering the low adherence to the three doses of vaccine against Hepatitis $B$, it is important to invest in the continuous supply of immunobiological and to expand orientation strategies regarding the importance of the complete vaccination scheme for this population.

\section{LB3.257 ESTIMATING PROGRESS TOWARDS ELIMINATION OF CONGENITAL SYPHILIS IN SOUTH AFRICA: INCIDENCE TRENDS USING CENTRALISED LABORATORY DATA, 2010-2015}

${ }^{1}$ Rudzani Mathebula, ${ }^{1}$ Lazarus Kuonza, ${ }^{1}$ Ranmini Kularatne, ${ }^{2}$ Tendesayi Chakezha. 1 National Institute for Communicable Diseases, Johannesburg, South African Republic; ${ }^{2}$ National Institute of Communicable Diseases, Johannesburg, South African Republic

\subsection{6/sextrans-2017-053264.492}

Introduction The elimination target for congenital syphilis (CS) is $<50$ cases per 100000 live births. We describe trends of laboratory data for possible early CS across South Africa (SA) and estimate its incidence in infants over a six year period.

Methods We conducted a cross-sectional analysis of laboratory data collected through the National Health Laboratory Service (NHLS) Corporate Data Warehouse (CDW) from 2010 to 2015. The NHLS CDW is a central repository of all laboratory tests performed in all public sector clinical laboratories in South Africa. We defined possible early CS as a positive serum Rapid Plasma Reagen (RPR) result in a child $<2$ years. Syphilis seropositivity rate was calculated as the number $<2$ years with a positive RPR test result divided by the total number $<2$ years tested during this time. Children with multiple RPR tests were counted once during this period. Incidence rate was calculated as the number of infants $<1$ year who had positive RPR results in a given year divided by the total number of livebirths for that year.

Results Of $84341<2$ years tested during 2010-2015 (92.3\% $<1$ year, $40.7 \%$ male), 6357 (7.5\%) tested positive. The number of children tested per year ranged from 23555 in Gauteng Province to 1555 in the Northern Cape. RPR seropositivity in $<2$ years increased from $6 \%$ in 2010 to $9 \%$ in 2015 (p-value $<0.001)$. Estimated national annual CS incidence rates in infants increased from 99/100,000 livebirths in 2010 to $119 / 100,000$ livebirths in 2015. Largest increases in incidence rates were KwaZulu-Natal (44-115/100,000 livebirths), Limpopo (10-25/100,000 livebirths), and Mpumalanga (26-67/100,000 livebirths), while declines were observed in Eastern Cape (278-178/100,000 livebirths), Northern Cape (168-66/100,000 livebirths) and Free State (84-51/100,000 livebirths).

Conclusion Though limited to laboratory results, the analysis shows high incidence possible CS. SA may need to intensify elimination efforts to reach the WHO target. Further clinical and maternal treatment information is needed in order to confirm incidence rates of early CS. 